\title{
Effects of chlordiazepoxide and reward magnitude on the acquisition and extinction of a partially reinforced response
}

\author{
JACK DEMAREST \\ Monmouth College, West Long Branch, New Jersey 07764 \\ and \\ JOHN R. MacKINNON \\ Connecticut College, New London, Connecticut 06320
}

\begin{abstract}
This study describes the relationship between the tranquilizing drug chlordiazepoxide HCL and different magnitudes of reward during acquisition and extinction of a partially reinforced runway response. The results do not support predictions drawn directly from Amsel's (1958, 1962) frustrative nonreward hypothesis. Animals exhibited the same magnitude of reward extinction effect whether sedated during acquisition or not. Some evidence was found of a reversal in this effect in animals sedated in both acquisition and extinction.
\end{abstract}

Hulse's (1958) study of the interaction of magnitude of reward and schedule of reinforcement is one of the main lines of evidence for Amsel's $(1958,1962)$ frustration hypothesis. He found that animals trained with small reward were more resistant to extinction than animals trained with large reward if every acquisition trial had been reinforced. With a partial schedule of reinforcement, however, animals receiving large reward in acquisition were more resistant to extinction than animals receiving small reward. These paradoxical results were replicated by Wagner (1961).

The interpretation of these results in terms of Amsel's $(1958,1962)$ frustration theory depends on the occurrence of anticipatory frustration $\left(\mathrm{r}_{\mathrm{F}}\right)$ under partial reinforcement in acquisition, and the counterconditioning of this aversive emotional response to approach behavior. Since anticipatory frustration and its associated stimulus $\left(\mathrm{s}_{\mathrm{F}}\right)$ presumably evoke a competing-disruptive response, continued approach behavior in the presence of $\mathrm{r}_{\mathrm{F}}-\mathrm{S}_{\mathrm{F}}$ reconditions $r_{F}-S_{F}$ to approach responses. In the situation produced by differential magnitudes of reward, animals trained under partial large reward learn to respond in the presence of greater $r_{F}-S_{F}$ than animals trained under partial small reward. The large-reward

This research was part of a Master's thesis submitted by the first author to the Department of Psychology, Connecticut College. An abbreviated version of this study was reported at the EPA meetings, Washington, D.C., May 1973. Reprints may be obtained from Jack Demarest, Department of Psychology, Monmouth College, West Long Branch, New Jersey 07764. animals would be expected to exhibit greater resistance to extinction than the partially reinforced small-reward animals. On the other hand, with continuous $(100 \%)$ reinforcement, the animals never experience nonreward and its associated anticipatory frustration $\left(\mathrm{r}_{\mathrm{F}}\right)$ in acquisition. The occurrence of nonreinforcement in extinction would produce the animals' first experience of aversive primary frustration. Primary frustration should also be most intense for the animals with the greatest expectancy of reward $\left(r_{G}\right)$. According to reward expectancy theory (Spence, 1956), the magnitude of $\mathrm{r}_{\mathrm{G}} \mathrm{s}_{\mathrm{G}}$ would be greater, at the end of acquisition, for large-reward animals than for small-reward animals. Therefore, acquisition training under continuous large reward should lead to faster extinction than continuous small-reward training.

Suppose we were able to eliminate the emotional consequences of nonreward? What would this do to the magnitude-of-reward effect? Amsel (Amsel \& Ward, 1965) has suggested that "the relative nonpersistence of large reward Ss under continuous reinforcement could also be shown in a partially rewarded group by reducing the anticipatory emotional consequence of nonreward in acquisition through the administration of a sedative or tranquilizer (p. 37).,"

If frustration can be eliminated in acquisition, animals should not show the typical magnitude effect for partially reinforced responding; in fact, there should be a reversal of this effect. Theoretically, animals that learned to run for small reward while under sedation should be more resistant to extinction than animals that learned to run for large reward 
while under sedation, even though trained under a partial schedule of reinforcement.

The present study examined the effects of chlordiazepoxide hydrochloride (CDP), a mild tranquilizing drug, on resistance to extinction of animals trained to either of two differential magnitudes of reward (500 vs. $45 \mathrm{mg}$ ) under a partial schedule of reinforcement. This drug was selected at the suggestion of Ison (Note 1) that the drug eliminated anticipatory emotional responses due to nonreinforcement but did not affect expectancy of reward. Expectancy of reward is needed in extinction for the occurrence of frustration and the elimination of the response (Amsel, 1962). The experimental procedure measured speed of running in a straight-alley runway and the perseverence of this response when reward was removed. The study was designed to test the prediction that the typical magnitude-of-reward effect under a partial reinforcement schedule (Hulse, 1958) could be reversed by the administration of chlordiazepoxide hydrochloride (CDP) in acquisition.

\section{METHOD}

\section{Subjects}

Sixty-four experimentally naive, male albino rats of the Charles River strain, approximately 90 days old at the beginning of pretraining, were used. They were run in two replications of 32 animals, each arriving in separate shipments.

\begin{abstract}
Apparatus
The apparatus was an enclosed, straight alley of the multirunway discrimination apparatus described in detail by MacKinnon (1967). The walls and floor of the alley and a metal food cup affixed to the end wall of the alley were painted flat black. A gray prestart area (PSB) and startbox (SB) were separated from each other by a metal guillotine-type door, and a clear Plexiglas door separated the SB from the alley proper. The length of the runway, from start door to food cup, was $1 \mathrm{~m}$.

The apparatus provided three measures of locomotor performance. Starting, running, and goal entry times over three successive $30-\mathrm{cm}$ segments of the runway were recorded by means of three Standard Electric timers, calibrated to $.01 \mathrm{sec}$, and operated by a system of photocells. A metal retrace door confined the animal in the food cup area. The guillotine doors were raised and lowered by a system of pulleys.
\end{abstract}

\section{Pharmacological Agent}

The drug used in this study, chlordiazepoxide hydrochloride (CDP), is a minor tranquilizer (perhaps better termed an anti- anxiety drug) supplied by Hoffman-LaRoche, Nutley, New Jersey.

The drug was dissolved into a solution, containing $10.0 \mathrm{mg}$ of CDP per $\mathrm{ml}$ of isotonic saline. The volume administered each animal was $0.1 \mathrm{ml}$ per $100 \mathrm{~g}$ of body weight. This dosage was established as the optimum for producing anxiety reduction in conflict situations while minimizing ataxia. Iwahara, Iwasaki, Nagamura, and Masuyama (1966), for example, noted that rats in a straight runway exhibited sluggish locomotor behavior and ataxia with dosages of $20 \mathrm{mg} / \mathrm{kg}$ of CDP but not with $10 \mathrm{mg} / \mathrm{kg}$.

\section{Experimental Design}

Acquisition and extinction performance was examined as a function of reward magnitude (large vs. small) and injection solution (CDP vs. isotonic saline). All animals were run under a $50 \%$ partial-reward schedule. Half the subjects received large reward (one 500-mg Noyes pellet) and half received a small reward (one 45-mg Noyes pellet). Half of the subjects in each magnitudeof-reward condition were administered intraperitoneal injections of CDP at a dosage of $10 \mathrm{mg} / \mathrm{kg}$ prior to each daily block of training trials, and half received an equal volume of isotonic saline. In extinction, half the subjects were maintained on the same drug condition and half were switched to the other solution (see Table 1). Control posttrial injections of the opposite drug solution were also administered, after the last trial of each day. The schedule of partial reinforcement for all groups in acquisition was determined by a Gellerman series, with the restriction that no more than two reward or noreward trials could occur in succession.

\section{Procedure}

Pretraining. Prior to the beginning of experimental training, the animals were housed individually and placed on an $11-\mathrm{g} /$ day deprivation schedule. This schedule was maintained throughout the experiment and included the amount of food consumed in the runway. Pretraining also involved systematic handling and habituation of the animals to the apparatus for 7 days. On the final 2 days of pretraining, each animal received an injection of isotonic saline, intraperitoneally, prior to exposure to the apparatus. In addition, the animals were introduced to the food pellet reward each day on a table in the same room as the apparatus. The animals were matched according to weight, and assigned to the four experimental acquisition groups on the final day of pretraining.

Acquisition and extinction. Each subject was run four trials a day for 16 days, a total of 64 acquisition trials. On each day of acquisition, an animal was given an intraperitoneal injection of drug or saline, replaced in the carrying cage, and tested $20 \mathrm{~min}$ after the injection. On each individual trial, the animal was retained in the SB for $2 \mathrm{sec}$, the SB door was then raised, starting the timing circuit, and the animal traversed the runway. When it entered the goalbox (GB), the GB door was lowered to prevent retracing. The animal was removed from the GB when all the food was eaten or, in the case of nonreward trials, after $20 \mathrm{sec}$, and returned to the carrying cage. The GB area was wiped clean with a damp sponge after each trial. The animals were run in

Table 1

Experimental Design of Drug and Magnitude of Reward Manipulations With a Schedule of Partial Reinforcement

\begin{tabular}{|c|c|c|c|c|c|}
\hline \multirow{2}{*}{$\begin{array}{c}\text { Reward } \\
\text { Condition }\end{array}$} & \multicolumn{2}{|c|}{ Acquisition Drug } & \multicolumn{2}{|c|}{ Extinction Drug } & \multirow{2}{*}{$\begin{array}{c}\text { Extinction } \\
\text { Group Designation }\end{array}$} \\
\hline & Pretest & Posttest & Pretest & Posttest & \\
\hline \multirow[t]{2}{*}{ Large Reward } & Drug & Saline & $\begin{array}{l}\text { Drug } \\
\text { Saline }\end{array}$ & $\begin{array}{l}\text { Saline } \\
\text { Drug }\end{array}$ & $\begin{array}{l}D-D(n=8) \\
D-S(n=8)\end{array}$ \\
\hline & Saline & Drug & $\begin{array}{l}\text { Drug } \\
\text { Saline }\end{array}$ & $\begin{array}{l}\text { Saline } \\
\text { Drug }\end{array}$ & $\begin{array}{l}\text { S-D }(n=8) \\
\text { S-S }(n=8)\end{array}$ \\
\hline \multirow[t]{2}{*}{ Small Reward } & Drug & Saline & $\begin{array}{l}\text { Drug } \\
\text { Saline }\end{array}$ & $\begin{array}{l}\text { Saline } \\
\text { Drug }\end{array}$ & $\begin{array}{l}\text { D-D }(n=8) \\
\text { D-S }(n=8)\end{array}$ \\
\hline & Saline & Drug & $\begin{array}{l}\text { Drug } \\
\text { Saline }\end{array}$ & $\begin{array}{l}\text { Saline } \\
\text { Drug }\end{array}$ & $\begin{array}{l}\text { S-D }(n=8) \\
\text { S-S }(n=8)\end{array}$ \\
\hline
\end{tabular}


squads of eight, each in rotation, and the order of running was randomized each day. After the first few days of acquisition, an animal completed all four trials within a period of 20-30 min, with an intertrial interval of approximately 5-8 min. For any subject, the time between the first injection and the last trial was no more than 40-50 min.

Extinction was identical to acquisition, with the exception that reward was omitted. The food cup remained in the GB during extinction and, since no animal failed to run to the goal area, the procedural details remained the same as for acquisition. Extinction trials were run for 9 days, for a total of 36 trials.

\section{RESULTS}

\section{Acquisition}

Latencies were converted to reciprocals to yield speed scores (i.e., distance traveled/sec). The mean median speeds for the last 20 trials of acquisition in each segment of the runway are shown in Table 2. These data for each segment were analyzed by means of a four-factor mixed design analysis of variance to determine if there were any effects of drug condition, reward magnitude, replications, or trials. The results indicated that there were no significant differences in start speeds for either the magnitude of reward or the drug manipulations. Analysis of the run segment indicated that there was a significant drug effect; the drug reduced the running speed of the animals $(p<.001)$. There was also a small, but significant, magnitude-of-reward effect; large-reward subjects ran faster than small-reward subjects $(p<.05)$. There was also a significant trials effect $(p<.001)$. In the goal segment, there was a significant drug effect $(p<.001)$, but the magnitude effect did not reach traditional levels of significance. The direction of the findings, however, was consistent with those in the run segment. All other effects failed to reach significance at the .05 level.

\section{Extinction}

Since the groups reached different asymptotic acquisition levels, it was necessary to correct extinction scores so that all extinction curves began at the same level. The data were transformed so that each extinction trial was represented as a proportion of its final asymptotic acquisition speed (Roberts, 1969; Wagner, 1961). The performances of each of the eight groups in the start and goal segments, plotted in blocks of four trials, are shown in Figure 1. The run measure was similar to those and will not be shown. A four-factor mixed design analysis of variance was computed to yield the general findings listed below. Subsequent two-factor mixed-design analyses were computed independently for each of the four drug conditions to assess the trend of the reward magnitude effect.

The general finding of the extinction phase is that the main effects of drug condition, magnitude of reward condition, and the interaction of the drug and
Table 2

Mean Median Speed $(\mathrm{cm} / \mathrm{sec})$ Through Each 30-cm Segment of the Runway Over the Final 20 Trials of Acquisition

\begin{tabular}{ccccrr}
\hline & \multicolumn{2}{c}{ Drug } & & \multicolumn{2}{c}{ Saline } \\
\cline { 2 - 3 } \cline { 5 - 6 } Segment & Large & Small & & \multicolumn{2}{c}{ Reward } \\
Start & 55.2 & 51.9 & & Small \\
Run & 92.1 & 86.7 & & 121.8 & 106.2 \\
Goal & 46.2 & 43.2 & & 54.6 & 51.0 \\
\hline
\end{tabular}

magnitude conditions were all highly significant. Animals extinguished under CDP were more resistant to extinction than those extinguished under saline $(\mathrm{p}<.001)$, and large-reward animals were generally more resistant to extinction than smallreward animals $(p<.001)$. However, these magnitude of reward results were shown to be dependent on the drug state of the animal $(p<.005)$. Animals extinguished under the influence of CDP did not show the typical magnitude-of-reward effect.

Control group (S-S). The performance of the animals trained and extinguished under saline in the start and goal segments of the runway is shown in the first frame of Figure 1. These data replicate the findings of Hulse (1958) and Wagner (1961) for partially rewarded subjects. Partially reinforced large reward was found to be more resistant to extinction than partially reinforced small reward $(\mathrm{p}<.05)$.

Chlordiazepoxide in acquisition but not extinction (D-S). The performance of animals trained under the influence of the drug and extinguished under saline is shown in the second frame of Figure 1. These data indicate that large-reward animals were significantly more resistant to extinction than small-reward animals $(p<.01)$. A significant reward-magnitude effect, however, appeared only in the start segment. Despite a similar trend in the goal segment and the run segment, neither the magnitude nor the Trials by Magnitude interactions reached significance at the .05 level. Overall, the D-S group was less resistant to extinction than any other drug condition $(p<.001)$.

Chlordiazepoxide in extinction but not acquisition (S-D). The start and goal segment running speeds for the animals trained with saline and extinguished under the influence of the drug are shown in the third frame of Figure 1. There was no difference in resistance to extinction between animals trained with large or small magnitude or reinforcement. There was a significant difference between this group's overall performance and the overall performance of the control group. Group S-D was more resistant to extinction than Group S-S $(p<.001)$.

Chlordiazepoxide in both acquisition and extinction (D-D). The start and goal speeds for the animals trained and extinguished under the influence of the 


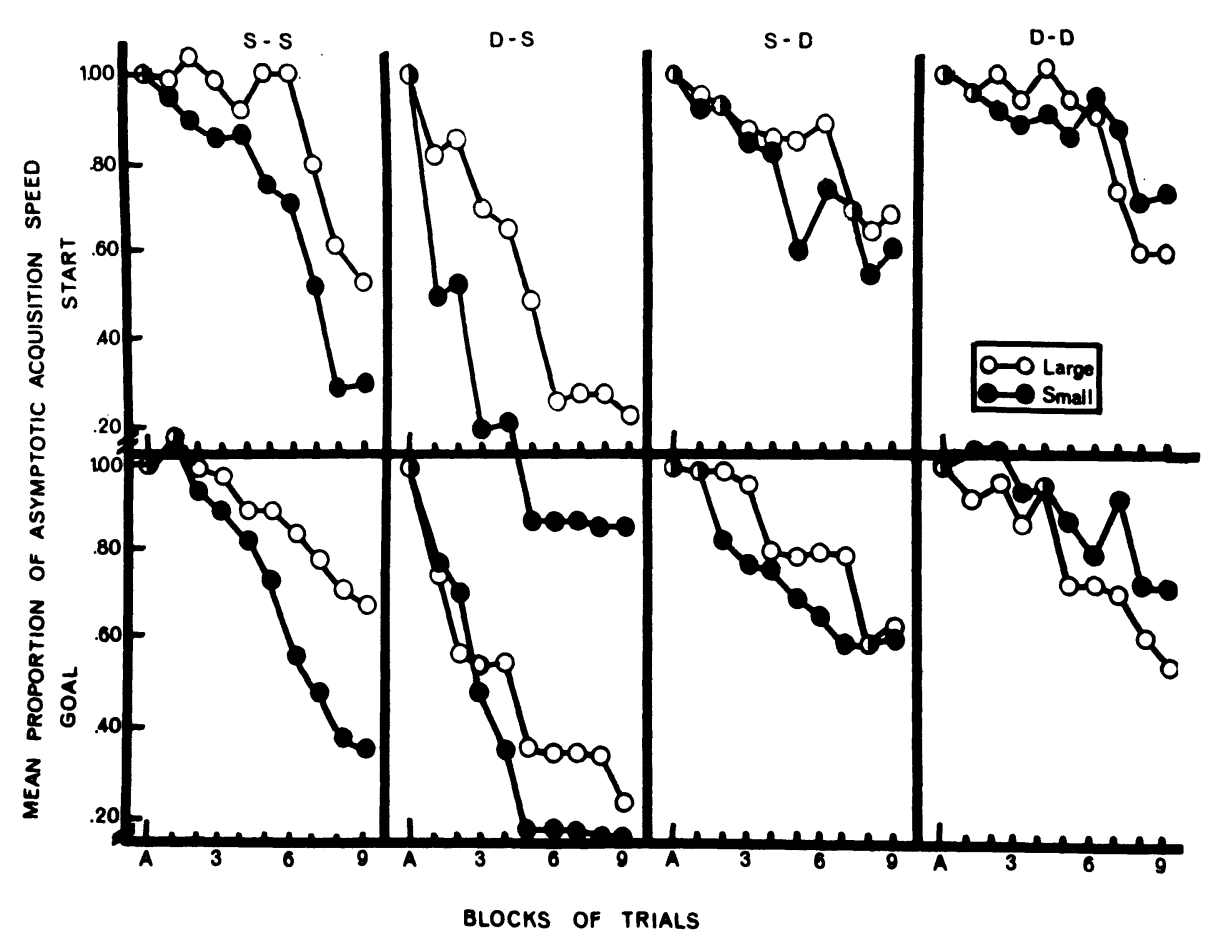

Figure 1. Start and goal speed in extinction represented as a proportion of each animal's final asymptotic acquisition speed. The data are separated into drug treatment categories and plotted in blocks of four trials. $D=$ tested after injection of drug; $S=$ tested after injection of saline. The first letter represents the acquisition drug condition; the second letter refers to the extinction drug condition.

drug are shown in the last frame of Figure 1. Though there was some suggestion of a reversal of the typical magnitude-of-reward effect, these differences did not approach acceptable levels of significance. There was, however, a significant Trials by Magnitude interaction in the start segment, indicating that the extinction slopes diverged $(p<.01)$. Analysis of the run segment revealed no significant magnitude or Trials by Magnitude effect, but did show a significant Trials by Magnitude by Replication interaction $(p<.005)$. This interaction revealed that the tendency for the small-reward animals to be more resistant to extinction than the large-reward animals was limited primarily to the first replication. There was no difference between the magnitude-of-reward groups in the second replication. Overall, there was a significant difference between the D-D group's performance and the overall performance of the control group. Group D-D was more resistant to extinction than Group S-S $(p<.001)$.

\section{DISCUSSION}

There are three dominant outcomes of this study, the magnitude-of-reward effect, the drug effect, and the interaction of these two variables.

The evidence provided by the acquisition data and by the extinction performance of the control group (i.e., Group S-S) indicate that the magnitudeof-reward variable has a strong influence on runway behavior. Its effect is to produce faster running in acquisition and greater resistance to extinction. These data are already well documented (Hulse, 1958; Wagner, 1961) and need no further discussion, other than to mention that the frustration account has been used to interpret these results.

The finding that pretrial injections of CDP lead to slower running times in acquisition and retarded extinction has also been previously reported (Iwahara, Iwasaki, Nagamura, \& Masuyama, 1966; Iwahara, Nagamura, \& Iwasaki, 1967). Similar results have been found using the pharmacologically related drugs amobarbital sodium (Rosen, Glass, \& Ison, 1967; Wagner, 1963) and ethanol (Nelson \& Wollen, 1965).

It is the interaction of these two variables, however, that we are primarily interested in in this study. The animals that were trained under the drug and extinguished under saline (D-S) represent the experimental condition postulated by Amsel and Ward (1965) to show a reversal of the usual magnitude-ofreward effect for partially rewarded animals. The data, however, give no indication of a reversal of the magnitude effect. Large-reward animals continued 
to persist in running longer than small-reward animals. Unexpectedly, the group trained and extinguished under the drug (D-D) did show some evidence of a reversal of the usual reward-magnitude effect. Though the main effect of reward size was not significant in this group, a Trials by Magnitude interaction was found to be significant. This interaction indicates that the large-reward animals extinguished running at a faster rate than the smallreward animals, at least in the start segment, in contrast to the slower rate of extinction for the largereward animals in the control group.

These results raise serious questions for accounts of extinction behavior based on the principle of frustrative nonreward. Frustration theory does not predict a reversal of the magnitude-of-reward effect in the D-D group, at least not to the same extent as would be expected in the D-S group. The absence of such an effect in the D-S group suggests that some other factor, perhaps peculiar to the drug itself, may enter into these results. It is interesting, in this light, that at least two previous studies have also failed to support frustration theory using the barbiturate, sodium amobarbitol. Ison and Pennes (1969) failed to find a predicted disruption of the partial reinforcement effect using this drug, and Gray (1969) found that this drug failed to alter the frustration effect. Further analysis of similar pharmacological manipulations of nonreward would appear to be useful in resolving this issue.

\section{REFERENCE NOTE}

1. Ison, J. R. Pharmacological approaches to two process learning theory. Paper presented at the meeting of the Behavioral Pharmacology Society, Groton, Connecticut, May 1968.

\section{REFERENCES}

AMSEL, A. The role of frustrative nonreward in noncontinuous reward situations. Psychological Bulletin, 1958, 55, 102-119.
AMSEL, A. Frustrative nonreward in partial reinforcement and discrimination learning: Some recent history and a theoretical extension. Psychological Review, 1962, 69, 306-328.

AMSEL, A., \& W ARD, J. S. Frustration and persistence: Resistance to discrimination following prior experience with the discriminanda. Psychological Monographs, 1965, 79(4, Whole No. 597).

GraY, J. A. Sodium amobarbital and effects of frustrative nonreward. Journal of Comparative and Physiological Psychology, 1969, 69, 56-64.

HulsE, S. H., JR. Amount and percentage of reinforcement and duration of goal confinement in conditioning and extinction. Journal of Experimental Psychology, 1958, 56, 48-57.

Ison, J. R., \& PenNes, E. S. Interaction of amobarbital sodium and reinforcement schedule in determining resistance to extinction of an instrumental running response. Journal of Comparative and Physiological Psychology, 1969, 68, 215-219.

Iwahara, S., Iwasaki, T., Nagamura, N., \& Masuyama, E. Effect of chlordiazepoxide upon partially reinforced behavior of rats in the straight runway. Japanese Psychological Research, 1966, 8, 131-135.

IWAhara, S., NAGAMUra, N., \& IwasakI, T. Effects of chlordiazepoxide upon experimental extinction in the straight runway as a function of partial reinforcement in the rat. Japanese Psychological Research, 1967, 9, 128-134.

MACKInNon, J. R. Interactive effects of the two rewards in a differential magnitude of reward discrimination. Journal of Experimental Psychology, 1967, 75, 329-338.

Nelson, P. B., \& Wollen, K. A. Effects of ethanol and partial reinforcement upon runway acquisition. Psychonomic Science, 1965, 3, 135-136.

RoBERTs, W. A. Resistance to extinction following partial and consistent reinforcement with varying magnitudes of reward. Journal of Comparative and Physiological Psychology, 1969, 67, 395-400.

Rosen, A. J., Glass, D. H., \& Ison, J. R. Amobarbitol sodium and instrumental performance changes following reward reduction. Psychonomic Science, 1967, 9, 129-130.

SPENCE, K. W. Behavior theory and conditioning. New Haven: Yale University Press, 1956.

W AGNER, A. R. Effects of amount and percentage of reinforcement and number of acquisition trials on conditioning and extinction. Journal of Experimental Psychology, 1961, 62, 234-242.

WAGNER, A. R. Sodium amytal and partially reinforced runway performance. Journal of Experimental Psychology, 1963, 65, 474-477.

(Received for publication August 4, 1977; accepted September 29, 1977.) 